\title{
Saranthe klotzschiana (Koer.) Eichl. (Marantaceae) e seu mecanismo explosivo de polinização ${ }^{1}$
}

\author{
EVELISE LOCATELLI ${ }^{2,3}$, ISABEL C. MACHADO² e PETRÚCIO MEDEIROS ${ }^{2}$
}

(recebido: 16 de outubro de 2003; aceito: 12 de agosto de 2004)

\begin{abstract}
Saranthe klotzschiana (Koer.) Eichl. (Marantaceae) and its explosive pollination mechanism). The pollination biology of Saranthe klotzschiana (Koer.) Eichl. was studied at an altitudinal forest ("brejo de altitude"), in the Parque Ecológico João Vasconcelos Sobrinho, Caruaru municipality - Pernambuco state, northeastern Brazil ( $8^{\circ} 18^{\prime} 36^{\prime \prime}$ S and 36 $00^{\circ} 00^{\prime \prime}$ ). Saranthe klotzschiana bears inflorescences with 2.5-5.5 cm in length, and ca. 10-30 flowers, measuring 6-10 $\mathrm{mm}$ in length. The anthesis is diurnal with flowers opening at 4:00 $\mathrm{h}$ and lasting up to 14:30 $\mathrm{h}$. Each inflorescence bears 4-12 open flowers per day. Each flower produced $1-3 \mu \mathrm{L}$ of nectar with sugar concentration ranging from $27 \%$ to $32 \%$. At dawn, frequent visits of diurnal moths and bees of the families Anthophoridae (Centris aenea, Epicharis (Epicharoides) sp., Mesoplia similis, Rhathymus acutiventris, and $R$. bicolor nigripes), Apidae (Euglossa truncata, E. carinilabris, Eulaema bombiformis, E. cingulata, E. nigrita, and Melipona scutellaris), and Colletidae (Ptiloglossa sp.) were observed. The bees visited flowers throughout anthesis, with a visiting peak from 6:00 to 11:00 a.m. Hummingbirds (Amazilia fimbriata, Chlorostilbon aureoventris, and Phaethornis ruber) were also observed visiting flowers of $S$. klotzschiana, beginning at 8:00 h. Before a visit the style is vertically positioned in relation to the ovary due to the tension played by the staminode. After a visit the style is detached, and the pollen is deposited in the proboscis (moth), tongue (bees) or beak (hummingbirds) of the visitor. The large bees and the hummingbirds were considered the main pollinators due to their efficacy on triggering the pollination mechanism.
\end{abstract}

Key words - bees, hummingbirds, Marantaceae, Northeastern Brazil, pollination

RESUMO - (Saranthe klotzschiana (Koer.) Eichl. (Marantaceae) e seu mecanismo explosivo de polinização). A biologia da polinização de Saranthe klotzschiana (Koer.) Eichl. foi estudada em uma Mata Serrana (brejo de altitude) no Parque Ecológico João Vasconcelos Sobrinho, Caruaru-PE ( $8^{\circ} 18^{\prime} 36^{\prime \prime}$ e $36^{\circ} 00^{\prime} 00^{\prime \prime}$ ). Saranthe klotzschiana apresenta inflorescências com 2,5-5,5 cm de comprimento, com ca. 10-30 flores, as quais medem 6-10 mm de comprimento. A antese é diurna, as flores abrem à partir das 4:00 $\mathrm{h}$ e fecham por volta das 14:30 h, abrindo cerca de 4-12 flores por inflorescência/dia. É produzido 1-3 $\mu \mathrm{L}$ de néctar por flor, com concentração de açúcares entre $27 \%$ e $32 \%$. Ao amanhecer, foram observadas visitas freqüentes de mariposas e de abelhas, das famílias Anthophoridae (Centris aenea, Epicharis (Epicharoides) sp., Mesoplia similis, Rhathymus acutiventris e $R$. bicolor nigripes), Apidae (Euglossa truncata, E. carinilabris, Eulaema bombiformis, E. cingulata, E. nigrita e Melipona scutellaris) e Colletidae (Ptiloglossa sp.). As abelhas visitaram as flores ao longo de todo o período de antese, sendo o pico de visitas das 6:00 h às 11:00 h. A partir das 8:00 h iniciavam as visitas dos beija-flores Amazilia fimbriata, Chlorostilbon aureoventris e Phaethornis ruber. Antes das visitas o estilete encontra-se em posição vertical em relação ao ovário, preso por tensão pelo estaminódio. Ao toque do visitante floral, o estilete se enrola, havendo deposição de pólen na probóscide (mariposa), língua (abelhas) ou no bico (beija-flores). As abelhas grandes e os beija-flores foram considerados os principais polinizadores devido à eficiência no desencadeamento do mecanismo de polinização.

Palavras-chave - abelhas, beija-flores, Marantaceae, Nordeste do Brasil, polinização

\section{Introdução}

A família Marantaceae apresenta distribuição pantropical sendo característica de florestas tropicais úmidas (Heywood 1978). Possui cerca de 31 gêneros e 800 espécies (Tomlinson 1969), das quais $80 \%$ são

1. Parte da Tese de Doutorado da primeira autora, desenvolvida junto ao Programa de Pós-Graduação em Biologia Vegetal da Universidade Federal de Pernambuco.

2. Universidade Federal de Pernambuco, Departamento de Botânica, Laboratório de Biologia Floral e Reprodutiva, Rua Professor Nelson Chaves s/n, 50372-970 Recife, PE, Brasil.

3. Autor para correspondência: eveliselocatelli@yahoo.com.br encontradas na América Tropical, 11\% na Ásia e 9\% na África (Kennedy 2000).

O gênero Saranthe é exclusivamente neotropical apresentando cerca de cinco a sete espécies no Brasil (Schumann 1902, Andersson 1998). No Nordeste brasileiro, os principais locais de ocorrência da família são as matas úmidas que se localizam na faixa costeira e em áreas de serras no interior, chamadas localmente de brejos de altitude (Arns 1997).

O mecanismo de polinização das espécies de Marantaceae é altamente especializado, caracterizado pela apresentação secundária de pólen e pelo movimento explosivo e irreversível do estilete, tendo sido Lindley (1826) o primeiro a descrevê-lo. 
Os trabalhos mais recentes de biologia floral com espécies de Marantaceae são os de Kennedy (1978, 2000), que abordou, respectivamente, algumas espécies de Calathea e a diversificação do mecanismo de polinização em Marantaceae. Classen-Bockhoff (1991) estudou a biologia floral de Thalia geniculata, registrando os movimentos florais desencadeados pelas visita do polinizador e Classen-Bockhoff \& Pischtschan (2000) realizaram estudos anatômicos do movimento explosivo do estilete.

No Novo Mundo, o maior grupo de polinizadores de espécies de Marantaceae é o das abelhas da tribo Euglossini. Essa tribo, restrita aos Neotrópicos, possui cinco gêneros (Aglae, Eufrisea, Euglossa, Eulaema e Exaerete), sendo que Euglossa e Eulaema são os polinizadores mais comuns, principalmente em espécies de Calathea (Kennedy 1978) e naquelas que apresentam corola com tubo curto, como as espécies de Mirosma, Ctenanthe e Stromanthe (Andersson 1981). De acordo com Kennedy (1978), o primeiro autor a citar visitas de abelhas Euglossini em Marantaceae foi Ducke, em 1910, estudando a apifauna do Pará, Brasil.

Apesar do relativo conhecimento sobre o mecanismo de polinização na família Marantaceae, até o momento nenhum trabalho detalhado sobre a biologia floral e a polinização foi realizado enfocando espécies ocorrentes na América do Sul. Neste trabalho são apresentadas a fenologia, a morfologia e a biologia floral, com ênfase no mecanismo de polinização de Saranthe klotzschiana (Koer.) Eichl., uma espécie ocorrente em vegetação de brejo de altitude, no Nordeste do Brasil.

\section{Material e métodos}

A população de Saranthe klotzschiana foi estudada de janeiro a abril de 1999 e 2000, no Parque Ecológico Vasconcelos Sobrinho ( $8^{\circ} 18^{\prime} 36^{\prime \prime} \mathrm{S}$ e $\left.36^{\circ} 00^{\prime} 00^{\prime \prime} \mathrm{W}\right)$, situado a $12 \mathrm{~km}$ do município de Caruaru, agreste de Pernambuco. O Parque, com área florestal de 354 ha, é uma das unidades de conservação de brejos de altitude sobre o maciço da Borborema. Localiza-se na Serra dos Cavalos, com uma altitude variando entre 800 e $900 \mathrm{~m}$.

Os brejos de altitude são áreas úmidas isoladas, localizadas nas zonas semi-áridas do agreste e do sertão nordestino (Andrade-Lima 1960). Essas áreas apresentam características peculiares, como: altitudes superiores a $600 \mathrm{~m}$, clima úmido ou sub-úmido e precipitação anual entre 900 e $1.300 \mathrm{~mm}$. Os brejos de altitude nordestinos apresentam vegetação natural coberta por floresta perenifólia ou subperenifólia, a qual recobre os topos e as vertentes de serras, circundadas, nas altitudes inferiores, por vegetação xerófila de caatinga (Andrade-Lima 1960, Andrade \& Lins
1964). Em virtude da umidade, há um gradiente vegetacional, no qual as espécies de caatinga vão sendo substituídas progressivamente por espécies de floresta semi-decídua (mata-seca), até o aparecimento da floresta perenifólia úmida (Sales et al. 1998).

Foram registradas informações sobre o desenvolvimento e modificações florais bem como sobre a formação de frutos em S. klotzschiana. A morfologia floral foi estudada a partir de material fresco conservado em álcool $70 \%$. Foram feitos fotos e desenhos das flores, observações sobre o tamanho, a cor e a duração da flor, o número de flores produzidas por inflorescência, o número e a disposição das peças florais, a disponibilidade de pólen, o horário e a seqüência de antese e da emissão de odor. A cor das flores foi determinada de acordo com o guia de cores de Smithe (1975). A receptividade do estigma foi testada com $\mathrm{H}_{2} \mathrm{O}_{2}$ (Zeisler 1938).

A quantidade de néctar produzido pelas flores abertas foi verificada utilizando-se micropipetas para extração e medidas do volume de néctar, em intervalos de 2 horas. Refratômetro de campo foi utilizado para estimar a concentração de néctar. Foram feitas 20 medidas de volume e concentração de néctar utilizando cinco flores de quatro indivíduos.

Para o estudo do sistema reprodutivo, botões em préantese foram isolados em sacos de organza, sendo feitos testes de polinizações controladas, consistindo de: 1) autopolinizações espontâneas, nas quais as flores ensacadas foram deixadas intactas para a verificação da formação ou não de fruto; 2) autopolinizações manuais, nas quais foram efetuadas polinizações com pólen da própria flor ou de outras flores do mesmo indivíduo (geitonogamia) nas flores previamente ensacadas; e 3) polinizações cruzadas, nas quais as flores ensacadas foram polinizadas com pólen oriundo de flores de indivíduos diferentes. Para a avaliação da eficiência de polinização natural (controle), flores foram marcadas e mantidas expostas, sendo verificado o número de frutos formados.

Para análise da fenologia reprodutiva e dos visitantes florais foram realizadas observações visuais diretas no campo, totalizando 280 horas de observação, em um agrupamento com mais de 30 indivíduos. Durante o período de desenvolvimento do estudo, 72 horas de observação, distribuídas em oito dias, entre os meses de janeiro e fevereiro/ 1999 e 2000, das 4:30 h às 14:00 h, foram dedicadas exclusivamente para o registro e observação dos visitantes quanto ao horário, a freqüência, a duração e seu comportamento às flores. Nesse período foi feita a classificação dos visitantes em raros, comuns e muito comuns, de acordo com a freqüência de visitas, tendo como critério o percentual obtido com as observações (de $0 \%$ a $5 \%$, de $6 \%$ a $15 \%$ e de $16 \%$ a $45 \%$, respectivamente). Alguns visitantes (abelhas e mariposas) foram capturados para posterior identificação por especialistas. Os beija-flores foram identificados por especialistas através de fotografias. Os indivíduos coletados foram conservados a seco e encontram- 
se na coleção do Laboratório de Biologia Floral e Reprodutiva da Universidade Federal de Pernambuco, como espécimestestemunho.

Material botânico foi coletado e encontra-se depositado no Herbário UFP - Professor Geraldo Mariz, Universidade Federal de Pernambuco (UFP12.318), como espécimetestemunho.

\section{Resultados}

A população estudada de Saranthe klotzschiana é constituída por cerca de 70 indivíduos, distribuídos ao longo de uma faixa de $50 \mathrm{~m}$ de largura e $700 \mathrm{~m}$ de comprimento, no interior da mata (figura 1). Saranthe klotzschiana tem porte herbáceo, medindo de 50 a $100 \mathrm{~cm}$ de altura (figuras 1,2). Apresenta padrão de floração e frutificação anual. O período de floração estende-se de janeiro a abril, com pico de floração em fevereiro e março. Frutos imaturos começaram a ser observados em fevereiro.

Saranthe klotzschiana apresenta inflorescências com cerca de 3 a $6 \mathrm{~cm}$ de comprimento e pedúnculo com ca. 4-10 cm de comprimento. Em cada inflorescência ocorrem de 8 a 30 címulas (cada uma abriga um par de flores) envolvidas por uma bractéola. Diariamente, em cada indivíduo, são encontradas 4 a 15 inflorescências compostas por 2-8 flores abertas, cada (figura 3). A abertura das flores inicia pela base da inflorescência. As flores são hermafroditas, possuem cálice com 3 sépalas de 3-5 mm de comprimento e corola com 3 pétalas membranáceas de $6-10 \mathrm{~mm}$ de comprimento de coloração rósea clara com o centro amarelado. $\mathrm{O}$ androceu é formado por dois estaminódios, um caloso com a parte distal petalóide e conspícua e outro cuculado o qual mantém o estilete sob pressão através de dois apêndices localizados na metade distal de sua base (figura 4A). Apresenta grãos de pólen brancos, os quais encontram-se aglutinados devido à presença de "pollenkitt". O gineceu apresenta ovário ínfero, uniovulado, estando a parte distal do estilete envolvida pelo capuz do estaminódio cuculado, preso sob tensão na posição ereta. No ápice do estilete existe uma depressão chamada de zona de aderência (Andersson 1981) ou depressão estilar (Kennedy 1978). É neste local que o pólen é depositado antes da antese.

A antese é diurna e inicia por volta das 4:00 h. As flores permanecem abertas até às 14:30 h. As pétalas são danificadas pelas visitas de alguns polinizadores, ficando as bordas murchas e escuras.

Durante toda a fase de antese da flor há produção de néctar que é acumulado na base da corola em pequenas quantidades, 1-3 mL por flor, sendo o volume médio de néctar por flor de $1,56 \mu \mathrm{L}( \pm 0,16 ; \mathrm{n}=20$ flores). A concentração de açúcares no néctar é, em média, $27,7 \%(+0,41 ; \mathrm{n}=20$ flores $)$, variando de $26 \%$ a $32 \%$. Não foi percebido odor nas flores.

Os resultados dos experimentos de polinização manual mostram que $S$. klotzschiana é autocompatível $(35 \%, \mathrm{n}=20)$, entretanto a produção de frutos resultantes dos experimentos de polinização cruzada foi mais elevada $(75 \%, \mathrm{n}=20)$ (tabela 1$)$.

O mecanismo de polinização de S. klotzschiana tem a seguinte sequiência (figura 4): os grãos de pólen

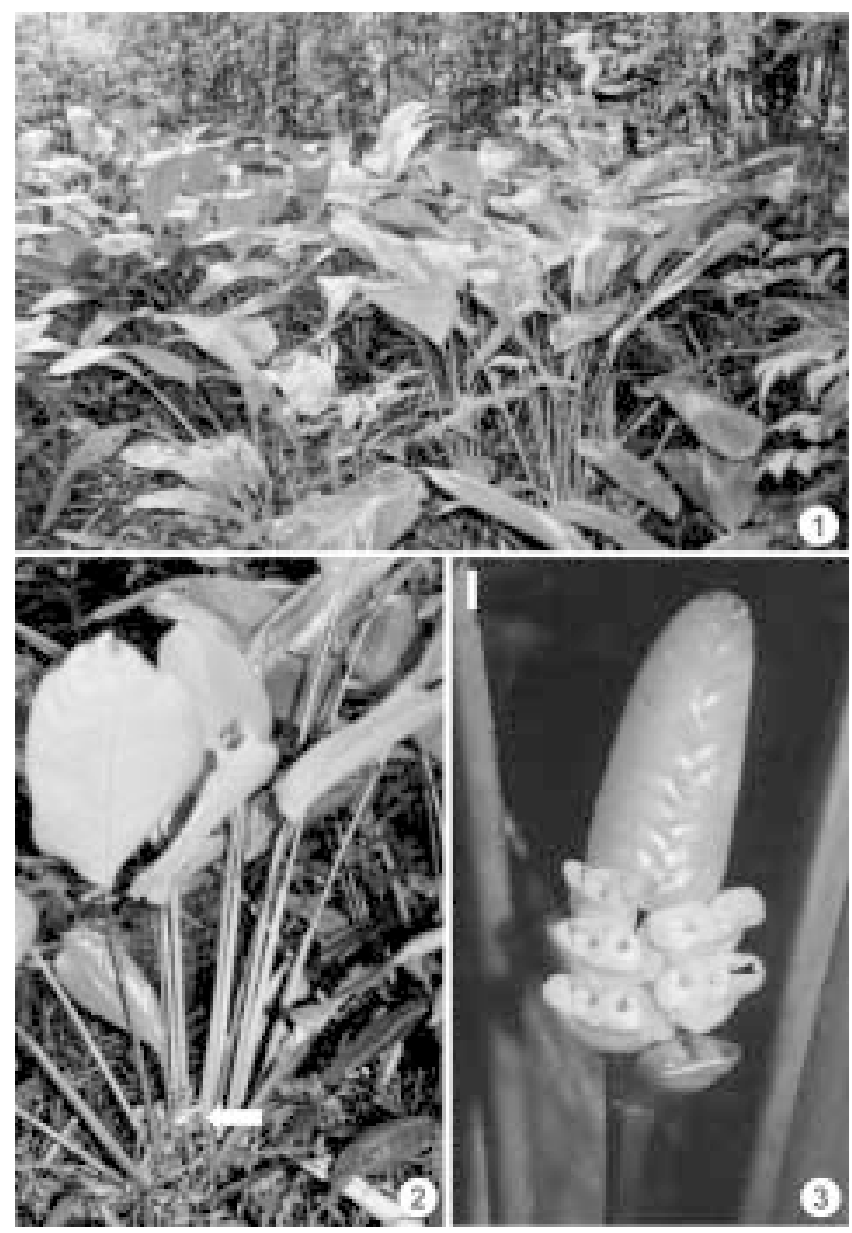

Figuras 1-3. Saranthe klotzschiana. 1. População estudada no brejo de altitude do Parque Ecológico João Vasconcelos Sobrinho, Caruaru, PE. 2. Indivíduo adulto (seta indica local das inflorescências) (aprox. $1 \mathrm{~m}$ altura). 3. Inflorescência (setas indicam o ápice do estilete) (Barra $=1 \mathrm{~cm})$.

Figures 1-3. Saranthe klotzschiana. 1. Studied population in an altitudinal forest ("brejo of altitude") in the Ecological Park João Vasconcelos Sobrinho, Caruaru, PE. 2. Adult individual (arrow shows inflorescence position) (approx. $1 \mathrm{~m}$ height). 3. Inflorescence (arrows show the style apex) $($ Bar $=1 \mathrm{~cm})$. 


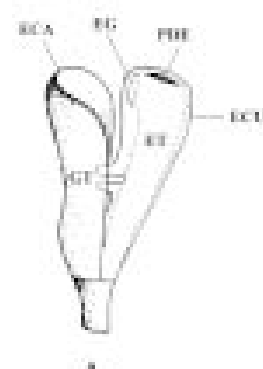

A
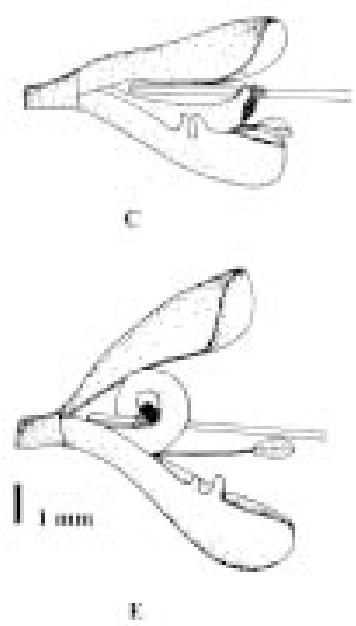

Figura 4. Esquema do mecanismo de desengatilhamento na flor de Saranthe klotzschiana. A. Vista frontal do androceu $(\mathrm{ECA}=$ estaminódio caloso; $\mathrm{EG}$ = estigma; $\mathrm{ET}$ = estilete; $\mathrm{ECU}=$ estaminódio cuculado; $\mathrm{GT}=$ gatilho; $\mathrm{PDE}=$ pólen na depressão estilar). B. Visitante insere a língua (ou bico) com pólen (PO: pólen) na flor à procura de néctar e toca no apêndice do estaminódio cuculado. C. Ocorre o desengatilhamento. O estilete move-se em direção a língua do visitante, tocando com o estigma na carga polínica que o mesmo carregava onde esta fica aderida. D. O estilete enrola-se contactando mais a língua (ou bico) do visitante. E. O movimento do estilete continua e agora é a depressão estilar carregada de pólen que contacta a língua (ou bico) do visitante. F. O visitante retira a língua (ou bico) de dentro da flor a qual agora fica com o pólen que estava na depressão estilar.

Figure 4. Scheme of the tripper mechanism in Saranthe klotzschiana flowers. A. Frontal view of the androecium $(\mathrm{ECA}=$ caloso staminodium; $\mathrm{EG}=$ stigma; $\mathrm{ET}$ : style; $\mathrm{ECU}=$ cuculate staminodium; $\mathrm{GT}=$ trigger $; \mathrm{PDE}=$ pollen in stylar depression). B. The visitant inserts the tongue (or bill) with pollen in flower to search the nectar and touches the appendices of the cuculate staminodium. C. Trippering. The style moves to the tongue of the visitor, and the stigma touches the pollen load carried by the visitor. D. The style twists and comes into contact with the tongue (or bill) of visitor better. E. The style movement continues and now is the stylar depression loaded with pollen which comes in contact with the tongue (or bill) of the visitor. F. The visitor removes the tongue (or bill) of the flower, which now keeps the pollen that was in the stylar depression.
Tabela 1. Resultados das polinizações controladas das flores de Saranthe klotzschiana, realizadas numa área de brejo de altitude do Parque Ecológico João Vasconcelos Sobrinho, Caruaru, PE.

Table 1. Controled pollinations in flowers of Saranthe klotzschiana, in an area of altitudinal forest ("brejo of altitude") in the Ecological Park João Vasconcelos Sobrinho, Caruaru, PE.

\begin{tabular}{lccc}
\hline Tratamento & $\begin{array}{c}\text { Flores } \\
\text { (n) }\end{array}$ & $\begin{array}{c}\text { Frutos } \\
\text { desenvolvidos } \\
\text { (n) }\end{array}$ & $\begin{array}{c}\text { Sucesso } \\
(\%)\end{array}$ \\
\hline Autopolinização espontânea & 20 & 7 & 35 \\
Autopolinização manual & 20 & 10 & 50 \\
Polinização cruzada & 20 & 15 & 75 \\
Controle (polinização aberta) & 30 & 25 & 83 \\
\hline
\end{tabular}

são depositados antes da antese na depressão estilar, momento em que o estilete ainda se encontra preso ao estaminódio cuculado (figura 4A). Quando o polinizador toca no apêndice existente ao lado desse estaminódio (figura 4B) acontece o desengatilhamento (figura 4C), sendo o estilete lançado para a frente (figura 4D), ficando totalmente curvo em posição oposta ao estaminódio caloso (figura 4E). Dessa maneira, o estigma é lançado em direção ao visitante e os grãos de pólen porventura oriundos de outra flor trazidos pelo visitante (figura 4B) ficarão depositados no estigma. Ao sair, o "pollenkitt" retido na depressão estilar será depositado sobre o visitante (figura 4F).

Os visitantes florais registrados em $S$. klotzschiana, foram (tabela 2): mariposas Aellopos titan (figura 8), abelhas das famílias Apidae [Euglossa truncata (figura 6); E. carinilabris; Eulaema bombiformes (figura 5), E. cingulata, E. nigrita, Melipona scutellaris]; Anthophoridae [Centris aenea; Epicharis (Epicharoides) sp. (figura 7); Mesoplia similis; Rhathymus acutiventris; R. bicolor nigripes], e Colletidae (Ptiloglossa sp.) e beija-flores Amazilia fimbriata; Chlorostilbon aureoventris (figura 9) e Phaethornis ruber (figura 10)]. Esses visitantes se sucederam temporalmente da seguinte forma: ao amanhecer, foram observadas visitas das mariposas e de abelhas desde o início da antese até às 9:00 h. As abelhas iniciavam as vistas às flores a partir das 5:00 h até seu fechamento, sendo o período de maior atividade entre 6:00 h e 11:00 h. A partir das 8:00 h iniciavam as visitas dos beija-flores que ficavam ativos nas flores até seu fechamento, sendo o período de maior atividade das 9:00 h às 13:00 h (figura 11). 
Tabela 2. Visitantes florais observados durante os meses de janeiro a abril de 1999 e 2000, em flores de Saranthe klotzschiana numa área de brejo de altitude do Parque Ecológico João Vasconcelos Sobrinho, Caruaru, PE. R = raro (até $5 \%$ das visitas); $\mathrm{C}=$ comum (6\% a $15 \%$ das visitas) e $\mathrm{MC}=$ muito comum (16\% a $45 \%$ das vistas $)$.

Table 2. Flower visitors of Saranthe klotzschiana observed from January to April/1999 and 2000, in area of altitudinal forest ("brejo of altitude") in the Ecological Park João Vasconcelos Sobrinho, Caruaru, PE. $\mathrm{R}=$ rare (up to $5 \%$ of visits); $\mathrm{C}=$ common ( $6 \%$ to $15 \%$ of visits) e $\mathrm{MC}=$ very common ( $16 \%$ to $45 \%$ of visits).

\begin{tabular}{|c|c|}
\hline Visitantes & $\begin{array}{l}\text { Freqüência de } \\
\text { visitas }\end{array}$ \\
\hline \multicolumn{2}{|l|}{ ABELHAS } \\
\hline \multicolumn{2}{|l|}{ ANTHOPHORIDAE } \\
\hline Centris aenea (Lepeletier, 1841) & $\mathrm{R}$ \\
\hline Epicharis (Epicharoides) spn & $\mathrm{R}$ \\
\hline Mesoplia similis Schrottky, 1920 & $\mathrm{R}$ \\
\hline Rhathymus acutiventris Friese, 1906 & $\mathrm{R}$ \\
\hline Rhathymus bicolor nigripes Friese, 1912 & $\mathrm{R}$ \\
\hline \multicolumn{2}{|l|}{ APIDAE } \\
\hline $\begin{array}{l}\text { Euglossa (Euglossa) truncata Rebelo \& } \\
\quad \text { Moure, } 1985\end{array}$ & $\mathrm{MC}$ \\
\hline $\begin{array}{l}\text { Euglossa (Glossurella) carinilabris } \\
\text { Dressler, } 1982\end{array}$ & $\mathrm{MC}$ \\
\hline Eulaema bombiforme Packard, 1869 & $\mathrm{C}$ \\
\hline Eulaema cingulata (Fabricius, 1804) & $\mathrm{MC}$ \\
\hline Eulaema nigrita (Lepeletier, 1841) & $\mathrm{MC}$ \\
\hline Melipona scutellaris Latreille, 1811 & $\mathrm{R}$ \\
\hline \multicolumn{2}{|l|}{ COLLETIDAE } \\
\hline Ptiloglossa sp. & $\mathrm{R}$ \\
\hline \multicolumn{2}{|l|}{ BEIJA-FLORES } \\
\hline \multicolumn{2}{|l|}{ TROCHILIDAE } \\
\hline Amazilia fimbriata Eleiot 1878 & $\mathrm{C}$ \\
\hline $\begin{array}{l}\text { Chlorostilbon aureoventris Bourcier \& } \\
\text { Mulsant } 1848\end{array}$ & $\mathrm{C}$ \\
\hline Phaethornis ruber Linnaeus 1758 & $\mathrm{MC}$ \\
\hline \multicolumn{2}{|l|}{ MARIPOSA } \\
\hline \multicolumn{2}{|l|}{ SPHINGIDAE } \\
\hline Aellopos titan Cramer, 1777 & $\mathrm{R}$ \\
\hline
\end{tabular}

Todas as abelhas apresentaram comportamento de visita semelhante, porém as mais freqüentes foram as espécies de Eulaema e Euglossa. A aproximação dessas abelhas às flores é frontal, apoiando-se em seguida com as pernas medianas e posteriores nas pétalas, inserindo então a língua na flor para a coleta de néctar. Nesse momento, os grãos de pólen ficavam aderidos na língua do inseto. Na maior parte das vezes as abelhas visitaram quase todas as flores abertas de uma mesma inflorescência, sendo sua permanência na inflorescência variável de acordo com o número de flores disponíveis. Em geral, permaneceram em cada flor de 2 a 7 segundos. Algumas vezes antes de se dirigirem a outra inflorescência, retrocederam em vôo pairado e transferiram parte do pólen para as corbículas das patas posteriores.

Entre os beija-flores, o mais freqüente foi Phaethornis ruber. De acordo com o modo de forragear todos os três beija-flores apresentaram comportamento semelhante. Forragearam em linhas de captura e, algumas vezes, foram territoriais, sendo que A. fimbriata foi o que mais apresentou este comportamento. Quando em linhas de captura os beijaflores visitaram as flores em intervalos constantes de 30 a 40 minutos. Os beija-flores pairaram defronte à flor e introduziram o bico, às vezes inserindo-o na mesma flor por duas a três vezes consecutivas. No decorrer da visita o pólen pode ficar aderido tanto na parte superior como na inferior do bico do beija-flor. De modo semelhante ao observado para as abelhas, os beija-flores visitaram a maior parte das flores abertas de uma mesma inflorescência. Suas visitas foram breves, em geral em vôo pairado, entretanto algumas vezes pousaram no escapo das inflorescências caídas que ficavam próximas às inflorescências que apresentavam flores abertas, a fim de coletar néctar.

\section{Discussão}

Saranthe klotzschiana possui padrão de floração do tipo anual, com floração intermediária (1-5 meses) (sensu Newstrom et al. 1994) e do tipo "steady-state" (sensu Gentry 1974). O fato de o período de floração ser relativamente longo com poucas flores abertas por dia por indivíduo, poderia reduzir a competição intraespecífica pelos serviços dos polinizadores, promovendo o deslocamento dos mesmos entre os indivíduos. Segundo Janzen (1971), espécies que abrem poucas flores por dia induzem os visitantes a apresentar comportamento de visita em linhas-de-captura, favorecendo o fluxo de pólen através da polinização cruzada. No caso dos beija-flores esse comportamento é também induzido devido à pequena quantidade de néctar oferecida por flor, o que os obriga a visitarem maior número de flores.

O pico de floração ocorreu na estação seca podendo trazer vantagens tanto para a planta como para as espécies visitantes, uma vez que a ocorrência de chuvas pesadas pode causar danos às flores e reduzir a frequiência de visitas (Janzen 1967). 

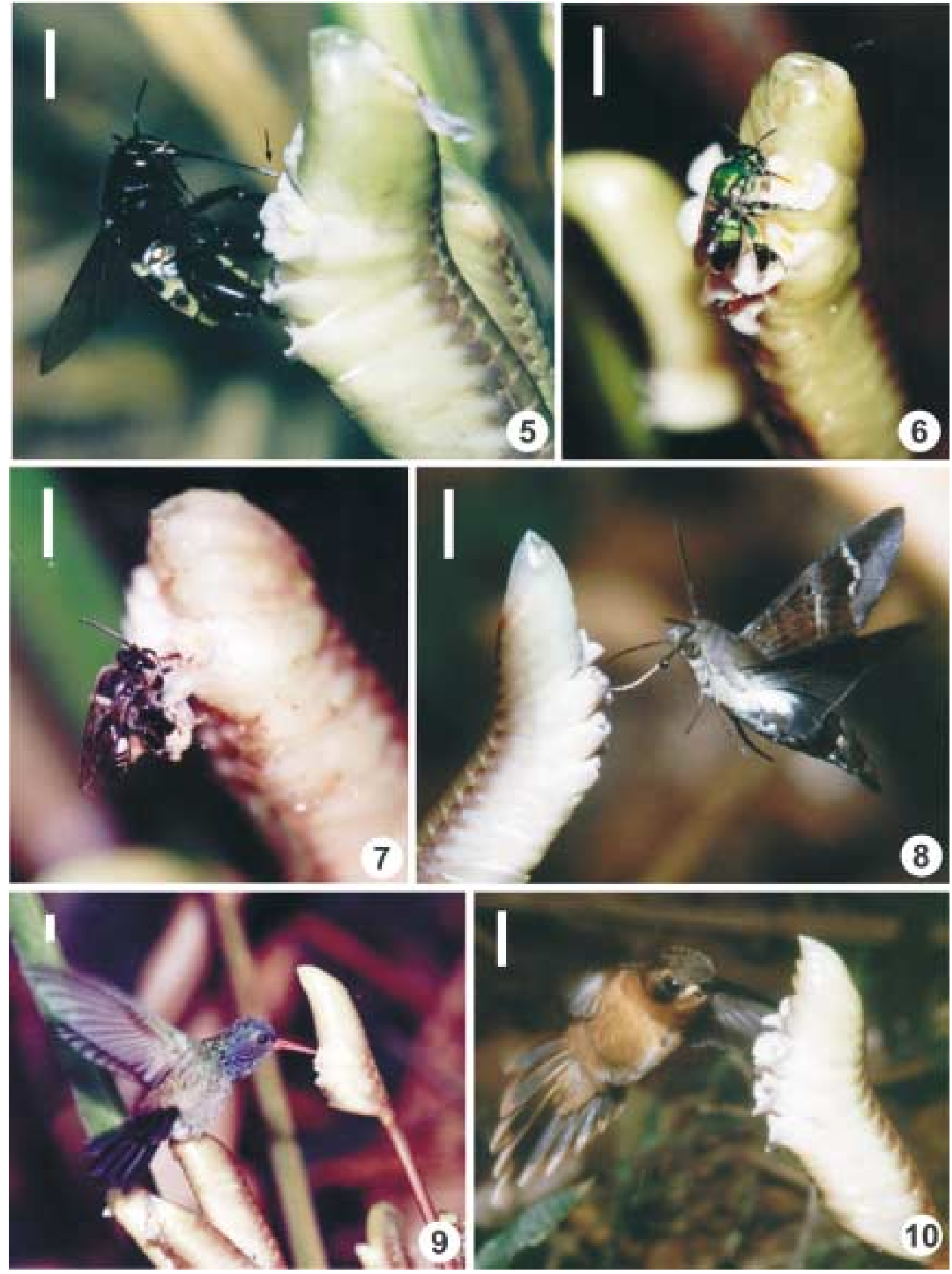

Figura 5-10. Visitantes florais de Saranthe klotzschiana em uma área de brejo de altitude do Parque Ecológico João Vasconcelos Sobrinho, Caruaru, PE. 5. Eulaema bombiformis. 6. Euglossa truncata. 7. Epicharis (Epicharoides) sp. 8. Aellopos titan. 9. Chlorostilbon aureoventris. 10. Phaethornis ruber (Barras $=1 \mathrm{~cm})$.

Figures 5-10. Flower visitors of Saranthe klotzschiana in an altitudinal forest ("brejo de altitude") in the "Ecological Park João Vasconcelos Sobrinho", Caruaru, PE. 5. Eulaema bombiformis. 6. Euglossa truncata. 7. Epicharis (Epicharoides) sp. 8. Aellopos titan. 9. Chlorostilbon aureoventris. 10. Phaethornis ruber $($ Bars $=1 \mathrm{~cm})$. 


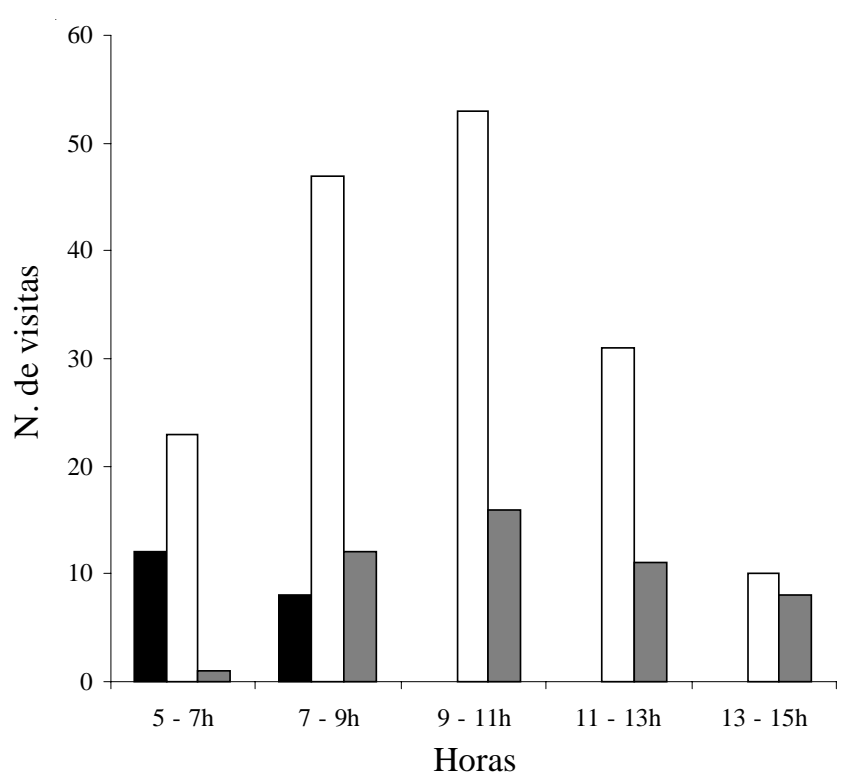

Figura 11. Média de freqüência dos visitantes florais de Saranthe klotzschiana em um dia de vista $(\mathrm{n}=8$ dias $)$ numa área de brejo de altitude no Parque Ecológico João Vasconcelos Sobrinho, Caruaru, PE. $\square=$ Mariposas; $\square=$ Abelhas; $\square=$ Beija-flores.

Figure 11. Average of de frequency of flower visitors in Saranthe klotzschiana in one day of visit ( $\mathrm{n}=8$ days) in an area of altitudinal forest ("brejo de altitude") in the Ecological Park João Vasconcelos Sobrinho, Caruaru, PE. $\quad$ = Moths; $\square=$ Bees; $\square=$ Hummingbirds.

Diversas características mencionadas por Faegri \& van der Pijl (1979) para a síndrome de melitofilia ocorrem nas flores de S. klotzschiana: simetria bilateral, contraste entre a cor das pétalas, róseo por fora e amarelo na entrada da corola, volume e concentração de açúcares do néctar. Além disso, os recursos florais oferecidos por S. klotzschiana (néctar e pólen) não estão expostos aos visitantes e a sua retirada depende de comportamento especializado. A coloração amarelada no centro da flor provavelmente funciona como guia de néctar fazendo com que o visitante, ao procurar o recurso, realize o desengatilhamento do estilete e, conseqüentemente, a polinização.

Os valores médios da concentração de açúcares no néctar das flores de $S$. klotzschiana indicam que não há especialização, sendo semelhantes aos valores apontados por Baker (1975) para flores visitadas tanto por abelhas solitárias (16\% - 50\%), borboletas $(13 \%-44 \%)$ como por beija-flores $(13 \%-30 \%)$. A concentração de açúcares no néctar também é próxima à de espécies ornitófilas estudadas por Percival (1974), Baker (1975) e Buzato et al. (2000), cujos valores estão entre $15 \%$ e $25 \%$. Essa sobreposição nos valores das concentrações de açúcares do néctar entre espécies polinizadas por abelhas solitárias, borboletas e beijaflores, provavelmente favorece a partilha de polinizadores.

O pólen só pode ser retirado se for desencadeado o mecanismo explosivo do estilete e o "pollenkitt" facilita sua aderência ao corpo do polinizador. Esse sistema permite que a produção de pólen seja minimizada, o que pode estar relacionado também ao fato da presença de apenas um ou três óvulos no ovário, comum em representantes dessa família (Yeo 1993).

Segundo os estudos anatômicos de ClassenBockhoff \& Pischtschan (2000) o movimento explosivo do estilete em espécies de Marantaceae é irreversível, portanto cada flor tem somente uma chance de ser polinizada. Kennedy (2000) afirma que a morfologia floral de Marantaceae é altamente especializada, uma vez que o pólen e o estigma são espacialmente separados e que, como o movimento estilar é irreversível, provavelmente essa posição pode evitar que algum pólen entre em contato com o próprio estigma, favorecendo a polinização cruzada.

Os resultados de polinização manual indicam que Saranthe klotzschiana é autocompatível. East (1940) menciona que a autocompatibilidade é uma regra nas famílias Marantaceae e Zingiberaceae. Entretanto, apesar de autocompatível, a taxa de polinização cruzada em S. klotzschiana foi bem mais alta do que a de autopolinização. Apesar de poder realizar autopolinização espontânea, seus atributos florais, principalmente os recursos oferecidos, atraem diversos tipos de visitantes (abelhas, beija-flores e mariposas), aumentando a possibilidade de polinização cruzada, importante para assegurar a variabilidade genética das populações e fundamental no caso de mudanças das condições ambientais (Richards 1986).

Kennedy $(1978,2000)$ cita que a autopolinização espontânea em Marantaceae pode ocorrer durante o processo de transferência do pólen ainda em botão e que, em muitas espécies, o que evita a entrada de seu próprio pólen no estigma é a forma do ápice do estilete e sua orientação em relação a antera. Uma leve mudança no ápice do estilete pode resultar na transferência de alguns grãos de pólen para a cavidade estigmática, os quais posteriormente podem germinar, resultando na autopolinização. Em Saranthe klotzschiana o ápice do estilete tem orientação oblíqua em relação à antera, semelhante ao descrito por Kennedy (2000) para outras espécies autogâmicas de Marantaceae. Essa característica do ápice do estilete, 
segundo esta autora, facilitaria a autopolinização, uma vez que durante o prolongamento do estilete alguns grãos de pólen poderiam cair no estigma (Kennedy 2000). Por sua vez em espécies alógamas dessa família, a extremidade estilar apresenta um ângulo mais ou menos reto com a antera, forçando todo o pólen a ir em direção à depressão estilar, o que dificulta a autopolinização.

De acordo com Kennedy (2000), um conjunto de características dos indivíduos e das flores de Marantaceae pode determinar a visita de poucos ou muitos tipos de polinizadores, tais como o comprimento da corola, a quantidade de néctar, o número de flores por inflorescência e a densidade de indivíduos da população. Em S. klotzschiana, o fato de haver oferta de uma grande quantidade de flores por dia devido à formação de densas manchas de indivíduos, bem como o tubo floral curto, provavelmente contribuem para a atração de um espectro maior de visitantes às flores. Para Baker (1961), a adaptação a diferentes tipos de polinizadores evolui gradualmente e está relacionada ao estabelecimento e à maior taxa de sobrevivência das espécies, frente a uma escassez ou falta dos polinizadores efetivos.

As abelhas Euglossini são citadas como os principais polinizadores de espécies de Marantaceae (Kennedy 1978), e dos cinco gêneros reconhecidos para esta tribo, os mais importantes na polinização das Marantaceae são Euglossa e Eulaema (Kennedy 1978). De fato, dentre as 11 espécies de abelhas registradas em $S$. klotzschiana, as principais polinizadoras foram espécies dos gêneros Eulaema e Euglossa. Além disso, segundo Gentry (1974), espécies com padrão de floração "steady-state" apresentam como principais grupos de polinizadores abelhas fêmeas da tribo Euglossini. Essas abelhas voam longas distâncias e estabelecem uma rota alimentar que favorece a polinização cruzada (Janzen 1971). Eulaema cingulata, E. nigrita e E. speciosa, bem como Euglossa spp. são citadas como polinizadores de espécies de Calathea em florestas úmidas (Kennedy 1978, Schemske \& Horvitz 1984).

As demais abelhas visitantes de Saranthe klotzschiana são polinizadores ocasionais devido à sua baixa freqüência. Ptiloglossa sp. visitou as flores de S. klotzschiana logo após a abertura da flor $(5: 00 \mathrm{~h})$, não sendo mais observada.

Todos os beija-flores registrados podem ser considerados polinizadores eficazes de S. klotzschiana, em virtude de seu comportamento e da freqüência de suas visitas. Apesar da já mencionada predominância de abelhas Euglossini como principais polinizadores de Marantaceae, beija-flores também foram ocasionalmente registrados visitando espécies dessa família (Kennedy 2000). Segundo Feinsinger \& Colwell (1978) alguns beija-flores podem transitar temporariamente entre as funções de linhas de captura e territorialidade como o observado em flores de S. klotzschiana. Provavelmente devido à baixa quantidade de néctar em flores de Saranthe, os beija-flores realizaram visitas em linhas de captura, neste caso, estabelecendo rotas mais ou menos regulares. Diferentes funções desenvolvidas pelos beija-flores são conhecidas em comunidades de Mata Atlântica no Sudeste do Brasil, sendo a disponibilidade do recurso alimentar um dos fatores que alteraria a escolha do tipo de comportamento alimentar dessas aves (Buzato et al. 1994, 2000, Sazima et al. 1996).

A única mariposa observada, Aellopos titan, por sua vez, foi considerada um polinizador ocasional, devido à baixa freqüência de visitas, bem como pelo fato de algumas vezes não desengatilhar o estilete, provavelmente devido à sua probóscide muito fina.

Dessa maneira, as abelhas Eulaema e Euglossa e os beija-flores foram considerados os principais polinizadores, tanto pela freqüência das visitas como pela eficiência no desencadeamento do mecanismo de polinização. Para esta espécie de Saranthe, que ocorre em matas úmidas muito fragmentadas, a utilização de diversos vetores de pólen é fundamental para o sucesso reprodutivo.

Agradecimentos - Aos Drs. Clemens Schlindwein, Danúncia Urban, João M.F. Camargo e Jesus Santiago Moure, pela identificação das abelhas; à Dra. Karla Norye pela identificação da planta; à MSc. Luciana Teixeira pelo acabamento artístico dos desenhos esquemáticos e aos dois assessores anônimos pelas valiosas sugestões. À Associação Plantas do Nordeste (PNE), ao Conselho Nacional de Desenvolvimento Científico e Tecnológico (CNPq), à Fundação O Boticário de Proteção à Natureza/MacArthur Foundation e ao Projeto Brejos de Altitude (MMA- BIRD-PROBIO) pelo apoio financeiro.

\section{Referências bibliográficas}

ANDERSSON, L. 1981. The neotropical genera of Marantaceae. - Circumscription and relationships. Nordic Journal Botany 1:218-245.

ANDERSSON, L. 1998. Marantaceae. In The families and genera of vascular plants, v.4, Flowering plants, Monocotyledons, Alismatanae and Commelinanae. (K. Kubitzki, ed.). Springer-Verlag, Berlin, p.278-293.

ANDRADE, G.O. \& LINS, R.C. 1964. Introdução ao estudo dos brejos pernambucanos. Arquivos do Instituto de Ciências da Terra, Recife 2:21-34. 
ANDRADE-LIMA, D. 1960. Estudos fitogeográficos de Pernambuco. Arquivo do Instituto de Pesquisa Agronômica, Secretaria da Agricultura, Indústria e Comércio, Recife, v.5, p.305-341.

ARNS, K.N.Y. 1997. Estudo taxonômico dos grupos Monotagma, Maranta e Myrosma (Marantaceae) em Pernambuco. Dissertação de mestrado, Universidade Federal de Pernambuco, Recife.

BAKER, H.G. 1961. The adaptation of flowering plants to nocturnal and crepuscular pollinators. The Quarterly Revue of Biology 36:64-73.

BAKER, H.G. 1975. Sugar concentrations in nectar from hummingbird flowers. Biotropica 7:37-41.

BUZATO, S., SAZIMA, M. \& SAZIMA, I. 1994. Pollination of three species of Abutilon (Malvaceae) intermediate between bat and hummingbird flower syndromes. Flora 189:327-334.

BUZATO, S., SAZIMA, M. \& SAZIMA, I. 2000. Hummingbird pollinated floras at three Atlantic forest sites. Biotropica 32:824-841.

CLASSEN-BOCKHOFF, R. 1991. Investigations on the construction of the pollination apparatus of Thalia geniculata (Marantaceae). Botanica Acta 104:183-193.

CLASSEN-BOCKHOFF, R. \& PISCHTSCHAN, E. 2000. The explosive style in Marantaceae preliminary results from anatomic studies. In Plant Biomechanics (H-Ch. Spat \& G. Sperl, eds.). Thieme-Verlag, New York, p.515-521.

EAST, E.M. 1940. The distribution of self-sterility in the flowering plants. Proceedings America Philosophical Society 82:449-518.

FAEGRI, K. \& VAN DER PIJL, L. 1979. The principles of pollination ecology. $3^{\text {rd }}$ ed. Pergamon Press, London.

FEINSINGER, P. \& COLWELL, R.K. 1978. Community organization among neotropical nectar-feeding birds. American Zoologist 18:779-795.

GENTRY, A.H. 1974. Flowering phenology and diversity in tropical Bignoniaceae. Biotropica 6:64-68.

HEYWOOD, V.H. 1978. Flowering plants of the world. Oxford University Press, Oxford.

JANZEN, D.H. 1967. Synchronization of sexual reproduction of trees within the dry season in Central America. Evolution 21:620-37.

JANZEN, D.H. 1971. Euglossine bees as long distance pollinators of tropical plants. Science 171:203-205.
KENNEDY, H. 1978. Systematic and pollination of the "closedflowered" species of Calathea (Marantaceae). University of California Publication in Botany 71:1-90.

KENNEDY, H. 2000. Diversification in pollination mechanisms in the Marantaceae. In Monocots. Systematics and evolution (K.L. Wilson \& D.A. Morrison, eds.). CSIRO Publishing, Collingwood, p.335-344.

LINDLEY, J. 1826. Calathea violacea. Botanical Register 12:t.962.

NEWSTROM, L.E., FRANKIE, G.W. \& BAKER, H.G. 1994. A new classification for plant phenology based on flowering patterns in lowland tropical rain forest trees at La Selva, Costa Rica. Biotropica 26:141-159.

PERCIVAL, M. 1974. Floral ecology of coastal scrub in southeast Jamaica. Biotropica 6:104-129.

RICHARDS, J.L. 1986. Plant breeding systems. George Allen \& Unwin, London.

SALES, M.F., MAYO, S.J. \& RODAL, M.J. 1998. Plantas vasculares das florestas serranas de Pernambuco: um checklist da Flora ameaçada dos Brejos de Altitude, Pernambuco, Brasil. Imprensa Universitária, Universidade Federal Rural de Pernambuco, Recife.

SAZIMA, M., BUZATO, S. \& SAZIMA, I. 1996. An assemblage of hummingbird-pollinated flowers in a montane forest in southeastern Brazil. Botanica Acta 109:149-160.

SCHEMSKE, D.W. \& HORWITZ, C.C. 1984. Variation among floral visitors in pollination ability: a precondition for mutualism specialization. Science 225:519-521.

SCHUMANN, K. 1902. Marantaceae. In Das Pflanzenreich (A. Engler, ed.). Engelmann, Berlin (Heft 11) 4:81-184.

SMITHE, F.B. 1975. Naturalist color guide. The American Museum of Natural History, New York

TOMLINSON, P.B. 1969. Classification of the Zingiberales (Scitamineae) with special reference to anatomical evidence. In Anatomy of the Monocotyledons (C.R. Metcalfe, ed.). Clarendon Press, Oxford, v.3, p.295-302.

YEO, P.F. 1993. Secondary pollen presentation: form, function and evolution. Plant Systematics and Evolution, Supplementum 6:1-268.

ZEISLER, M. 1938. Über die Abgrenzung der eigentlichen Narbenfläche mit Hilfe von Reaktionen. Beihifte zum Botanische Zentralblatt 58:308-318. 